\title{
Esophageal Synovial Sarcoma
}

National Cancer Institute

\section{Source}

National Cancer Institute. Esophageal Synovial Sarcoma. NCI Thesaurus. Code 695624.

A synovial sarcoma that affects the esophageal wall. 